\title{
The Balakrishnan Alpha Skew Truncated Cauchy Distribution with Applications in Modelling Currency Exchange Rate
}

(Pencongan Alfa Balakrishnan Taburan Cauchy Terpenggal dengan Aplikasi dalam Pemodelan Pertukaran Kadar Matawang)

\author{
SRICHARAN Shah, PARTHA Jyoti HaZARIKA* \& SUbrata CHAKRABORTY
}

\section{ABSTRACT}

In this article, a new class of alpha skew truncated Cauchy distribution is proposed by considering Balakrishnan methodology and investigated some of its related distributions. This article also derive various properties of this distribution along with its moments. The proposed distribution is applied in modeling currency exchange rate data with some other existing distributions, viz., skewed Cauchy distribution, skewed truncated Cauchy distribution, truncated skew Cauchy uniform distribution, doubly truncated skew Cauchy distribution, and alpha skew truncated Cauchy distribution. Based on the model selection criteria, Akaike Information Criteria (AIC) and Bayesian Information Criteria (BIC), the proposed distribution has shown significant improvement in modeling exchange rate dataset.

Keywords: Alpha-skew distribution; alpha-skew-truncated-Cauchy distribution; Balakrishnan alpha-skew-normal distribution; bimodal distribution; skew distribution

\section{ABSTRAK}

Dalam makalah ini, kelas baharu taburan Cauchy terpenggal pencong alfa dicadangkan dengan mempertimbangkan kaedah Balakrishnan dan beberapa taburan yang berkaitan dikaji. Makalah ini juga menerbitkan pelbagai sifat taburan bersama dengan momennya. Taburan yang dicadangkan digunakan dalam pemodelan data kadar pertukaran mata wang bersama dengan beberapa taburan sedia ada, iaitu, taburan Cauchy terpencong, taburan Cauchy terpenggal pencong, taburan seragam Cauchy terpenggal pencong, taburan Cauchy terpenggal pencong dua kali, dan taburan Cauchy terpenggal pencong alfa. Berdasarkan kriteria pemilihan model, Kriteria Maklumat Akaike (AIC) dan Kriteria Maklumat Bayesian (BIC), taburan yang dicadangkan mempunyai peningkatan yang signifikan dalam pemodelan set data kadar pertukaran.

Kata kunci: Taburan bimodal; taburan Cauchy terpenggal pencong alfa; taburan normal pencong alfa Balakrishnan; taburan pencong alfa; taburan pencong

\section{INTRODUCTION}

Skewed symmetric distributions starting from Azzalini (1985) have been researched by many statistical researchers in the last few years (for details see Chakraborty and Hazarika 2011). The skew Cauchy distribution (Arnold \& Beaver 2000) is one of the well-known skew symmetric distributions with the probability density function (pdf) given by

$$
f(z)=\frac{1}{\pi\left(1+z^{2}\right)}\left\{1+\frac{2}{\pi} \arctan (\alpha z)\right\} ; z \in R, \alpha \in R .
$$

However, due to the lack of finite moments this distribution suffers from limited applicability in real life.
To overcome this drawback, Nadarajah and Kotz (2007) introduced the truncated skew Cauchy distribution with pdf

$$
f(z)=\frac{1}{2 \arctan (h)\left(1+z^{2}\right)}\left\{1+\frac{\arctan (\alpha z)}{\arctan (h)}\right\} ;-h<z<h, \alpha \in R .
$$

Furthermore, the distribution in (2) can be extended to a doubly truncated skew Cauchy distribution with the following pdf as

$$
f_{D T C}(z ; A, B)=\frac{2(\arctan (\alpha z)-\arctan (A))}{[\arctan (B)-\arctan (A)]^{2}\left(1+z^{2}\right)} ; A<z<B, \alpha \in R, A<B .
$$

For the construction of skew-symmetric distributions starting from a symmetric (about 0) pdf $h($.$) , Huang$ and Chen (2007) introduced the concept of skew 
function $G($.$) , a Lebesgue measurable function such$ that, $0 \leq G(z) \leq 1$ and $G(z)+G(-z)=1, z \in R$, almost everywhere with the following pdf

$$
f(z)=2 h(z) G(z) ; z \in R .
$$

In 2016, applying the construction in (4), a new truncated skew Cauchy distribution was derived by Ashani et al. (2016), where $h(z)$ is the pdf of truncated Cauchy distribution and $G(z)$ is the cumulative distribution function (cdf) of uniform distribution in (4). The pdf of this new distribution (known as truncated skew Cauchy uniform distribution) is given by

$$
f(z)=\frac{\alpha z+h}{2 h \arctan (h)\left(1+z^{2}\right)} ;-h<z<h, \alpha \in R .
$$

Elal-Olivero (2010) developed a new form of skew distribution, known as the alpha skew normal distribution, which includes unimodal as well as bimodal behavior and the pdf is given by

$$
f(z ; \alpha)=\left(\frac{(1-\alpha z)^{2}+1}{2+\alpha^{2}}\right) \varphi(z) ; z \in R, \alpha \in R,
$$

where, $\varphi(z)$ is the pdf of standard normal distribution.

Using Elal-Olivero (2010) methodology, Hazarika and Chakraborty (2016) introduced the alpha skew truncated Cauchy distribution (two sided) with the pdf

$$
f_{A S T C}(z ; \alpha)=\left(\frac{(1-\alpha z)^{2}+1}{D_{(A, B)}\left(1+z^{2}\right)}\right) ; \quad A \leq z \leq B, \alpha \in R
$$

where, $D_{(A, B)}$ is a normalizing constant.

Besides this, distribution in (6) has attracted many researchers including Alhamide et al. (2019), Hazarika et al. (2020), and Shah et al. (2020a, 2020b).

In this article the main aim was to propose another class of alpha skew truncated Cauchy distribution by considering Balakrishnan (2002) methodology which is flexible enough to support unimodal as well as bimodal behaviors and some of its distributional properties are investigated. This distribution possesses finite moments of all orders and removes the drawback of skew Cauchy distribution namely, the lack of finite moments. Therefore, this distribution could be a better model for certain practical situations. One such situation in economics is discussed with the modeling finance exchange rate data of the United Kingdom Pound to the United States Dollar from 1800 to 2003.

The rest of this article is organized as follows. In the next section, we introduce a new alpha skew truncated Cauchy $B A S T C(\alpha)$ distribution and discuss some of its important distributional properties. A real-life application of this distribution is shown in subsequent section. Final conclusion regarding this work is provided in the last section.

\section{THE BALAKRISHNAN ALPHA SKEW TRUNCATED CAUCHY DISTRIBUTION}

In this section we define the Balakrishnan alpha skew truncated Cauchy distribution and discuss its basic properties as follows,

Definition 2: If a random variable $Z$ has the pdf

$$
f_{B A S T C}(z ; \alpha)=\frac{\left[(1-\alpha z)^{2}+1\right]^{2}}{C_{(A, B)}\left(1+z^{2}\right)} ; \quad A \leq z \leq B, \alpha \in R .
$$

Then, it is said to be the Balakrishnan alpha skew truncated Cauchy distribution with parameters $\alpha, A$ and $B$. In the rest of this article we shall refer the distribution in (8) as $B A S T C(\alpha)$. The normalizing constant $C_{(A, B)}$ is obtained as:

$$
C_{(A, B)}=\int_{A}^{B} \frac{\left[(1-\alpha z)^{2}+1\right]^{2}}{\left(1+z^{2}\right)} d z
$$

$=\int_{A}^{B} \frac{4-8 \alpha z+8 \alpha^{2} z^{2}-4 \alpha^{3} z^{3}+\alpha^{4} z^{4}}{\left(1+z^{2}\right)} d z$

$$
\begin{aligned}
= & \int_{A}^{B} \frac{4}{\left(1+z^{2}\right)} d z-\int_{A}^{B} \frac{8 \alpha z}{\left(1+z^{2}\right)} d z+\int_{A}^{B} \frac{8 \alpha^{2} z^{2}}{\left(1+z^{2}\right)} d z-\int_{A}^{B} \frac{4 \alpha^{3} z^{3}}{\left(1+z^{2}\right)} d z+ \\
& \int_{A}^{B} \frac{\alpha^{4} z^{4}}{\left(1+z^{2}\right)} d z
\end{aligned}
$$$$
=4(\arctan (B)-\arctan (A))-8 \alpha\left(\frac{\log \left[\left(1+B^{2}\right) /\left(1+A^{2}\right)\right]}{2}\right)+
$$$$
8 \alpha^{2}(B-A+\arctan (A)-\arctan (B))-
$$

$$
\begin{aligned}
& 4 \alpha^{3}\left(\frac{B^{2}-A^{2}+\log \left(1+A^{2}\right)-\log \left(1+B^{2}\right)}{2}\right)+ \\
& \alpha^{4}\left(\frac{B^{3}-A^{3}+3 A-3 B+3 \arctan (B)-3 \arctan (A)}{3}\right)
\end{aligned}
$$

On simplifying we get the result as

$$
\begin{aligned}
= & \left(4-8 \alpha^{2}+\alpha^{4}\right)(\arctan (B)-\arctan (A))+\frac{1}{3} \alpha((B-A) \\
& \alpha\left(24-6 B \alpha-3 \alpha^{2}+A^{2} \alpha^{2}+B^{2} \alpha^{2}+\right. \\
& \left.A \alpha(-6+B \alpha))+6\left(-2+\alpha^{2}\right) \log \left(\left(1+B^{2}\right) /\left(1+A^{2}\right)\right)\right)
\end{aligned}
$$


PROPERTIES OF $B A S T C(\alpha)$

- If $\alpha=0$, then we get $f(z)=\frac{1}{\left(1+z^{2}\right)(\arctan (B)-\arctan (A))}$.

- If $\alpha \rightarrow \pm \infty$, then we get $f(z)=$

$$
\frac{3 z^{4}}{\left(1+z^{2}\right)\left(B^{3}-A^{3}+3 A-3 B-3 \arctan (A)+3 \arctan (B)\right)} \text {. }
$$

- If $Z \sim B A S T C(\alpha)$, then $-Z \sim B A S T C(-\alpha)$.
For the symmetric interval $(-h, h)$, the pdf of $\operatorname{BASTC}(\alpha)$ distribution can be obtained as:

$$
f(z ; \alpha)=\frac{\left[(1-\alpha z)^{2}+1\right]^{2}}{C\left(1+z^{2}\right)} ;-h \leq z \leq h, \alpha \in R
$$

where, $C=\frac{2}{3} h \alpha^{2}\left(24+\alpha^{2}\left(-3+h^{2}\right)\right)+2\left(4-8 \alpha^{2}+\alpha^{4}\right) \arctan$ (h).

\section{PLOTS OF THE PDF}

The pdf of $B A S T C(\alpha)$ for different choices of the parameters $\alpha, A$ and $B$ are plotted in Figures 1 and 2.

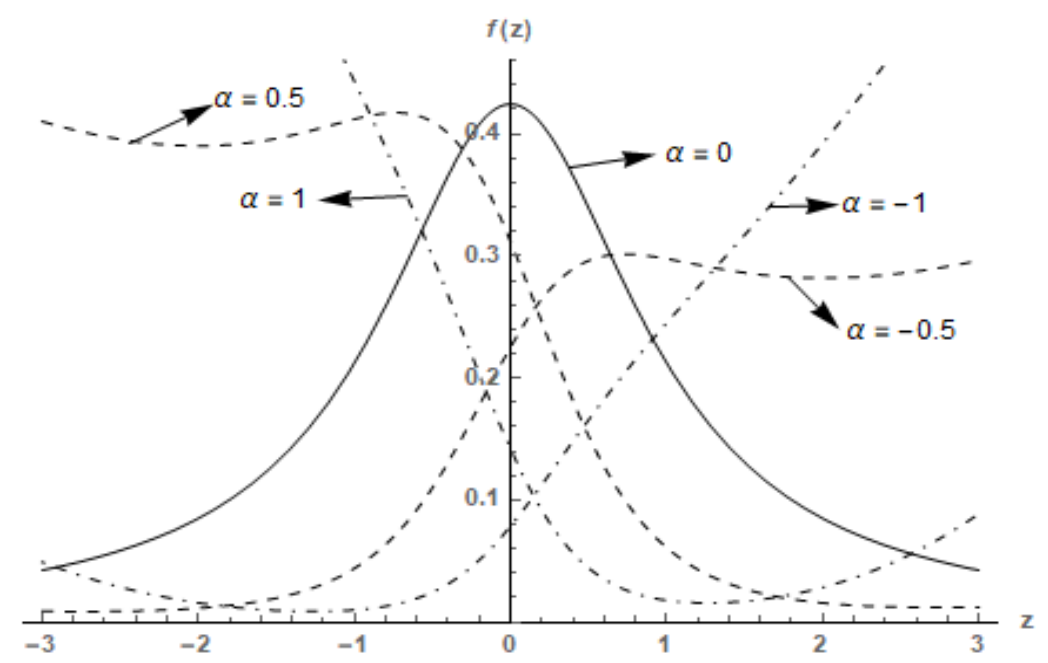

FIGURE 1. Plots of pdf for different choices of parameter $\alpha$ and fixed $A=-2$ and $B=3$

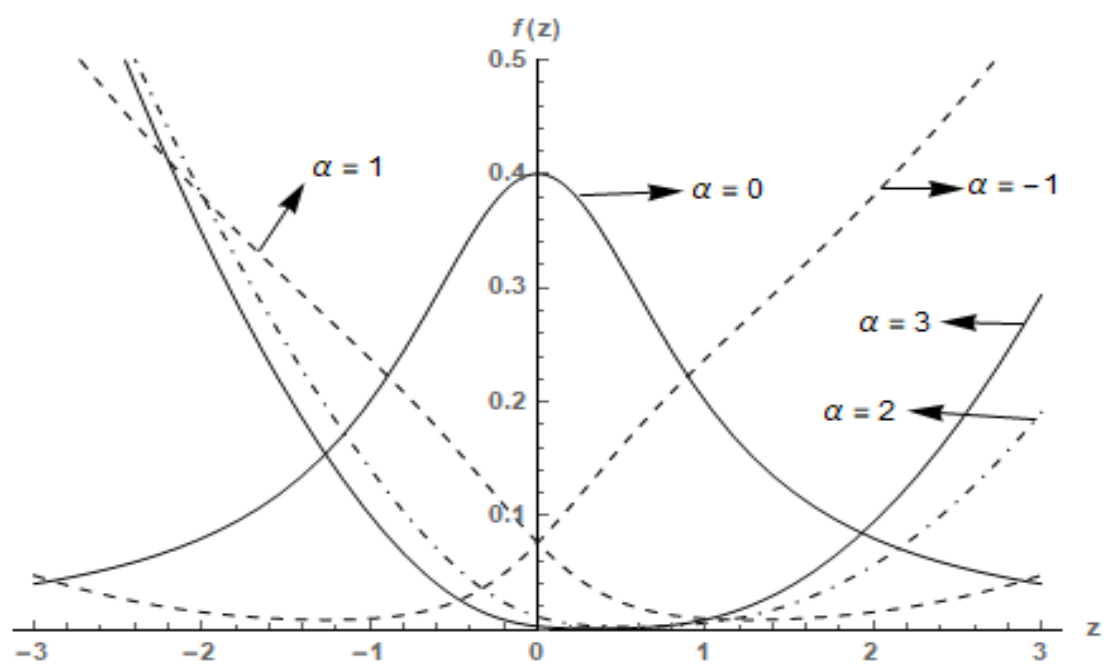

FIGURE 2. Plots of pdf for different choices of parameter $\alpha$ and fixed $A=-3$ and $B=3$ 
From Figures 1 and 2, it can be seen that the distribution has at most two modes as in the case of other alpha skew distributions (Elal-Olivero 2010; Hazarika \& Chakraborty 2014). Here, we may consider $\alpha$ as a scale parameter which impact number of modes, A and B refers to the truncation limits and are estimated by minimum and maximum order statistics.

Theorem 1: The cdf of $B A S T C(\alpha)$ distribution is given by

$$
F(t)=P(Z \leq t)=\frac{C_{(A, t)}}{C_{(A, B)}}
$$

where $C_{(A, t)}=\left(4-8 \alpha^{2}+\alpha^{4}\right)(\arctan (t)-\arctan (A))+$

$$
\begin{aligned}
& \frac{1}{3} \alpha\left(( t - A ) \alpha \left(24-6 t \alpha-3 \alpha^{2}+A^{2} \alpha^{2}+t^{2} \alpha^{2}+\right.\right. \\
& \left.A \alpha(-6+t \alpha))+6\left(-2+\alpha^{2}\right) \log \left(\left(1+t^{2}\right) /\left(1+A^{2}\right)\right)\right)
\end{aligned}
$$

Proof: See Appendix A.

Corollary 1: In particular, for large values of $\alpha \rightarrow \pm \infty$, $F(t)$ in Equation (9) reduces to

$$
F(t)=\frac{3 A-A^{3}-3 t+t^{3}-3 \arctan (A)+3 \arctan (t)}{3 A-A^{3}-3 B+B^{3}-3 \arctan (A)+3 \arctan (B)}
$$

\section{PLOTS OF THE CDF}

The cdf is plotted in Figure 3 for studying variation in its shape with respect to the parameter $\alpha$.

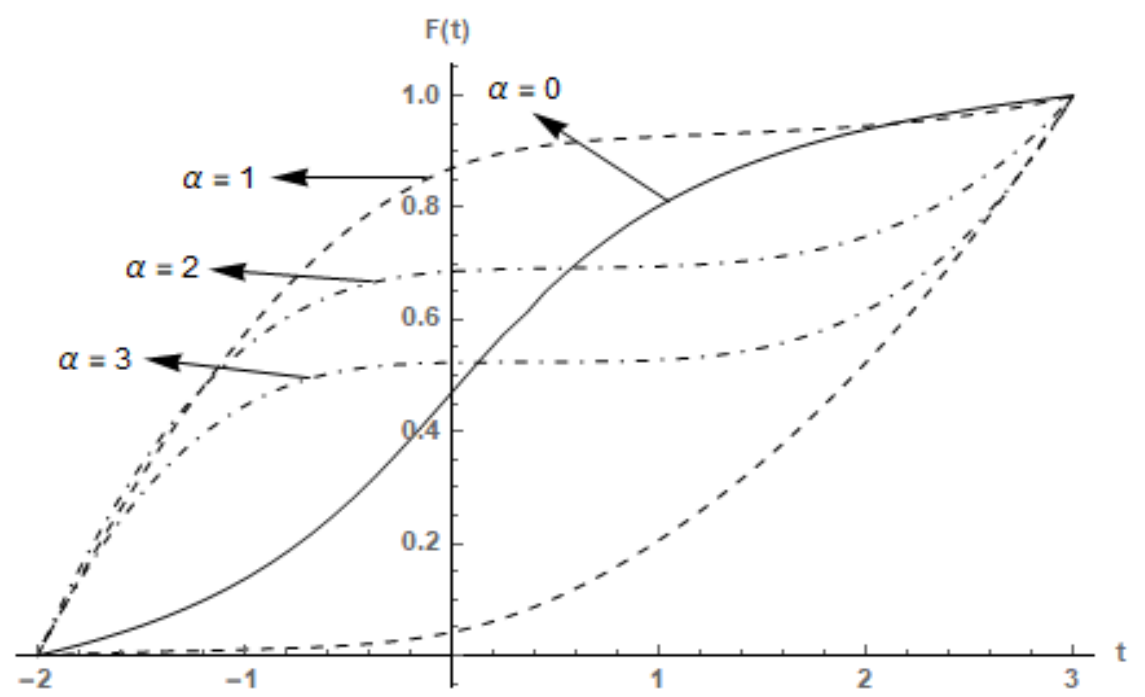

FIGURE 3. Plots of cdf for different choices of parameter $\alpha$ and fixed $A=-2$ and $B=3$

\section{MOMENTS}

Theorem 3: The $n^{\text {th }}$ order moment of BASTC $(\alpha)$ distribution is given by

$$
\begin{aligned}
E\left(Z^{n}\right)= & \frac{1}{C_{(A, B)}}\left[4\left\{I_{n}(B)-I_{n}(A)\right\}-8 \alpha\left\{I_{n+1}(B)-I_{n+1}(A)\right\}+\right. \\
& \cdot 8 \alpha^{2}\left\{I_{n+2}(B)-I_{n+2}(A)\right\}-4 \alpha^{3}\left\{I_{n+3}(B)-\right. \\
& \left.\left.I_{n+3}(A)\right\}+\alpha^{4}\left\{I_{n+4}(B)-I_{n+4}(A)\right\}\right]
\end{aligned}
$$

where $I_{n}(Z)$ is the Gauss hypergeometric function (Prudnikov et al. 1986) and it can be written as

$$
I_{n}(Z)=\int_{0}^{z} \frac{y^{n}}{1+y^{2}} d y=\frac{Z^{n+1}}{n+1^{2}} F_{1}\left(1, \frac{n+1}{2} ; \frac{n+3}{2} ;-z^{2}\right) .
$$

Proof:

$$
\begin{aligned}
E\left(Z^{n}\right)= & \frac{1}{C_{(A, B)}} \int_{A}^{B} \frac{z^{n}\left[(1-\alpha z)^{2}+1\right]^{2}}{\left(1+z^{2}\right)} d z \\
= & \frac{1}{C_{(A, B)}}\left[4 \int_{A}^{B} \frac{z^{n}}{\left(1+z^{2}\right)} d z-8 \alpha \int_{A}^{B} \frac{z^{n+1}}{\left(1+z^{2}\right)} d z+8 \alpha^{2} \int_{A}^{B} \frac{z^{n+2}}{\left(1+z^{2}\right)} d z\right. \\
& \left.-4 \alpha^{3} \int_{A}^{B} \frac{z^{n+3}}{\left(1+z^{2}\right)} d z+\alpha^{4} \int_{A}^{B} \frac{z^{n+4}}{\left(1+z^{2}\right)} d z\right] \\
& =\frac{1}{C_{(A, B)}}\left[4 G_{n}-8 \alpha G_{n+1}+8 \alpha^{2} G_{n+2}-4 \alpha^{3} G_{n+3}+\alpha^{4} G_{n+4}\right]
\end{aligned}
$$


Now, $G_{n}$ can be expressed as $G_{n}=\int_{0}^{B} \frac{z^{n}}{\left(1+z^{2}\right)} d z-\int_{0}^{A} \frac{z^{n}}{\left(1+z^{2}\right)} d z$ $=I_{n}(B)-I_{n}(A)$.

Similarly, $G_{n+1}=I_{n+1}(B)-I_{n+1}(A)$, $G_{n+2}=I_{n+2}(B)-I_{n+2}(A), G_{n+3}=I_{n+3}(B)-I_{n+3}(A)$ and $G_{n+4}=I_{n+4}(B)-I_{n+4}(A)$ and putting this results in (11), we get the desired result in (10).

From the equation of $n^{\text {th }}$ order moment, we get the first four moments by putting $n=1,2,3,4$ which is given in Appendix B. Though the expressions of the moments are too complicated this can be easily found out numerically. The plot of $E(Z)$ and $\operatorname{Var}(Z)$ are shown in Figure 4 for fixed values of $A=-2$ and $B=3$.
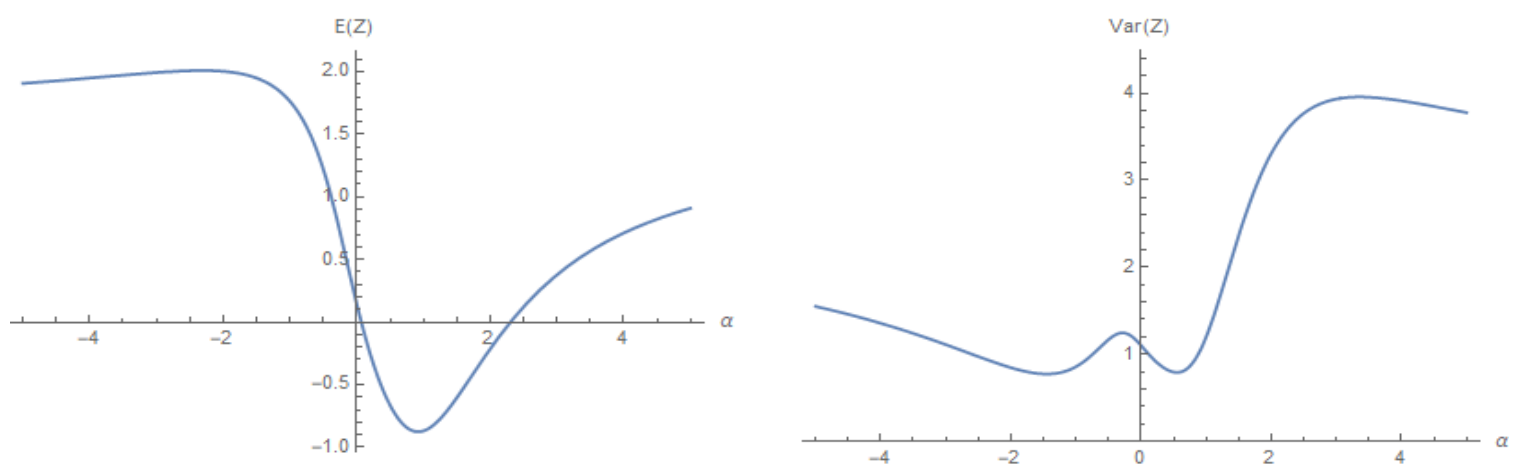

FIGURE 4. Plot of Mean and Variance for fixed $\mathrm{A}=-2$ and $\mathrm{B}=3$

REAL LIFE APPLICATIONS: GLOBAL FINANCIAL DATA

For illustration, here we consider the exchange rate data of the United Kingdom Pound to the United States Dollar from 1800 to 2003 (for details about dataset see Nadarajah and Kotz, 2007). For the common practice for exchange rate data, we transformed the values first by taking logarithms and then computing the relative changes from 1 year to the next (for details see Ashani et al. 2016). The summary statistic of the transformed data is given in Table 1. We then fitted the known distributions such as the skewed Cauchy distribution of Arnold and Beaver (2000), the truncated skew Cauchy distribution of Nadarajah and Kotz (2007), the doubly truncated skew Cauchy distribution, the truncated skew Cauchy uniform distribution of Ashani et al. (2016), $\operatorname{ASTC}(\alpha, A, B)$ distribution of Hazarika and Chakraborty (2016) and the proposed $B A S T C(\alpha, A, B)$ distribution to the transformed data by the method of maximum likelihood. For the present dataset the truncation limit for the distributions was chosen as $A=-0.606$ and $B=1.481$. While for the symmetrical truncation, the limit is 1.481. The AIC and BIC obtained in Table 2 have been used for model comparison. The following estimates were obtained and shown in Table 2.

TABLE 1. Summary Statistic of the data

\begin{tabular}{ccccc}
\hline Min. & Median & Mean & Max. & SD \\
\hline-0.6062926 & -0.0003246 & 0.0040339 & 1.4809978 & 0.1470195 \\
\hline
\end{tabular}

TABLE 2. MLE's, AIC, and BIC for the exchange rate data of the United Kingdom Pound to the United States Dollar from 1800 to 2003

\begin{tabular}{lccccccc}
\hline \multicolumn{1}{c}{ Distributions } & $\hat{h}$ & $\hat{A}$ & $\hat{B}$ & $\hat{\alpha}$ & $\log L$ & AIC & BIC \\
\hline Skew Cauchy & -- & -- & -- & 0.268 & -235.489 & 472.979 & 476.292 \\
Truncated Skew Cauchy & 1.481 & -- & -- & 0.187 & -139.066 & 282.133 & 288.759 \\
Doubly Truncated Skew Cauchy & -- & -0.606 & 1.481 & 0.112 & -156.104 & 318.207 & 328.147 \\
Truncated Skew Cauchy uniform & 1.481 & -- & -- & 0.577 & -139.060 & 282.227 & 288.253 \\
$\operatorname{ASTC}(\alpha, A, B)$ & -- & -0.606 & 1.481 & 0.763 & -66.1967 & 138.393 & 148.333 \\
$\operatorname{BASTC}(\alpha, A, B)$ & -- & -0.606 & 1.481 & 0.508 & $\mathbf{- 6 1 . 4 5 9}$ & $\mathbf{1 2 8 . 9 1 9}$ & $\mathbf{1 3 8 . 8 7 3}$ \\
\hline
\end{tabular}


From the Table 2, it is observed that the proposed distribution provides better fitting in terms of log-likelihood, AIC, and BIC. Thus, we say that this $\operatorname{BASTC}(\alpha, A, B)$ distribution turns out to be better choice for the exchange rate data of the United Kingdom Pound to the United States Dollar from 1800 to 2003 as compared to the other known related distributions considered here.

We have also fitted $B A S T C(\alpha, A, B)$ distribution by considering the subsets of the same dataset for the periods 1800-1900 and 1900-2003 and observed that average relative changes in the exchange rate for the 1800-1900 data is near zero while for 1900-2003 and 1800-2003 both use approximately 0.11 . From this it may be concluded that during early years i.e., from 18001900 the exchange rate was stable with not much relative change as compared to the later period.

\section{CONCLUSION}

In this study a new form of alpha skew truncated Cauchy distribution has been introduced and investigated some of its basic properties. Also, it possesses finite moments of all orders. Lastly, an application of the data fitting with a real life Global Financial data set regarding exchange rate has shown that the proposed distribution is far better as compared to the skewed Cauchy distribution, the skewed truncated Cauchy distribution, the truncated skew Cauchy uniform distribution, the doubly truncated skew Cauchy distribution, and the alpha skew truncated Cauchy distribution. Economic aspects of the finding of this work may be taken up for further investigation in a future project.

\section{REFERENCES}

Alhamide, A.A., Ibrahim, K., Alodat, M.T. \& Zin, W.Z.W. 2019 Bayesian inference for linear regression under alpha-skewnormal prior. Sains Malaysiana 48(1): 227-235.

Arnold, B.C. \& Beaver, R.J. 2000. The Skew-Cauchy distribution. Statistics \& Probability Letters 49(3): 285-290.

Ashani, Z.N., Bakar, M.R.A., Ibrahim, N.A. \& Adam, M.B. 2016. A skewed truncated Cauchy uniform distribution and its moment. Modern Applied Science 10(7): 174-182.
Azzalini, A. 1985. A class of distributions which includes the normal ones. Scandinavian Journal of Statistics 12(2):171178.

Balakrishnan, N. 2002. Discussion on "Skew multivariate models related to hidden truncation and/or selective reporting" by B.C. Arnold and R. J. Beaver. Test 11: 37-39.

Chakraborty, S. \& Hazarika, P.J. 2011. A survey of the theoretical developments in univariate skew normal distributions. Assam Statistical Review 25(1): 41-63.

Elal-Olivero, D. 2010 . Alpha-skew-normal distribution. Proyecciones (Antofagasta) 29(3): 224-240.

Hazarika, P.J. \& Chakraborty, S. 2016. Application of alpha skew truncated Cauchy distribution in exchange rate data. Journal of Statistical Distributions and Applications 2(2): 22-26.

Hazarika, P.J. \& Chakraborty, S. 2014. Alpha-skew-logistic distribution. IOSR Journal of Mathematics 10(4): 36-46.

Hazarika, P.J., Shah, S. \& Chakraborty, S. 2019. Balakrishnan alpha skew normal distribution: Properties and applications. Malaysian Journal of Science 39(2): 71-91

Huang, W.J. \& Chen, Y.H. 2007. Generalized Skew-Cauchy distribution. Statistics \& Probability Letters 77(11): 11371147.

Nadarajah, S. \& Kotz, S. 2007. A skewed truncated Cauchy distribution with applications in economics. Applied Economics Letters 14(13): 957-961.

Prudnikov, A.P., Brychkov, I.A. \& Marichev, O.I. 1986. Integrals and Series: Special Functions. Amsterdam: Gordon and Breach Science Publishers.

Shah, S., Chakraborty, S. \& Hazarika, P.J. 2020a. The Balakrishnan alpha skew logistic distribution: Properties and applications. International Journal of Applied Mathematics and Statistics 59(1): 76-92.

Shah, S., Hazarika, P.J. \& Chakraborty, S. 2020b. A new alpha skew laplace distribution: Properties and its applications. International Journal of Agricultural and Statistical Sciences 16(1): $1-10$

Department of Statistics

Dibrugarh University

Dibrugarh, Assam, 786004

India

*Corresponding author; email: parthajhazarika@gmail.com

Received: 29 August 2019

Accepted: 17 March 2020 


\section{APPENDIX A: PROOF OF CDF}

$F(t)=P(Z \leq t)=\frac{1}{C_{(A, B)}} \int_{A}^{t} \frac{\left[(1-\alpha z)^{2}+1\right]^{2}}{\left(1+z^{2}\right)} d z=$

$\frac{1}{C_{(A, B)}} \int_{A}^{t} \frac{(\arctan (t)-\arctan (A))\left[(1-\alpha z)^{2}+1\right]^{2}}{(\arctan (t)-\arctan (A))\left(1+z^{2}\right)} d z$

$=\frac{(\arctan (t)-\arctan (A))}{C_{(A, B)}}\left[4-\int_{A}^{t} \frac{8 \alpha z}{(\arctan (t)-\arctan (A))\left(1+z^{2}\right)} d z+\right.$

$\int_{A}^{t} \frac{8 \alpha^{2} z^{2}}{(\arctan (t)-\arctan (A))\left(1+z^{2}\right)} d z-$

$\left.\int_{A}^{t} \frac{4 \alpha^{3} z^{3}}{(\arctan (t)-\arctan (A))\left(1+z^{2}\right)} d z+\int_{A}^{t} \frac{\alpha^{4} z^{4}}{(\arctan (t)-\arctan (A))\left(1+z^{2}\right)} d z\right]$

$=\frac{(\arctan (t)-\arctan (A))}{C_{(A, B)}}\left[4-\int_{A}^{t} 8 \alpha z f_{D T C}(z ; A, t ; 0,1) d z+\int_{A}^{t} 8 \alpha^{2} z^{2} f_{D T C}\right.$

$(z ; A, t ; 0,1) d z-$

(A1)

$\left.\int_{A}^{t} 4 \alpha^{3} z^{3} f_{D T C}(z ; A, t ; 0,1) d z+\int_{A}^{t} \alpha^{4} z^{4} f_{D T C}(z ; A, t ; 0,1) d z\right]$

The result in (9) follows by putting the following results of the first four moments of doubly truncated skew Cauchy distribution (given below) in (A1).

$$
\begin{aligned}
& E\left[Z_{D T C}\right]=\frac{\log \left(1+t^{2}\right)-\log \left(1+B^{2}\right)}{2(\arctan (t)-\arctan (A))} \\
& E\left[Z^{2}{ }_{D T C}\right]=\frac{\arctan (t)-\arctan (A)-A+t}{\arctan (t)-\arctan (A)}, \\
& E\left[Z^{3}{ }_{D T C}\right]=\frac{\log \left(1+A^{2}\right)-\log \left(1+t^{2}\right)-A^{2}+t^{2}}{2(\arctan (t)-\arctan (A))}, \\
& E\left[Z^{4}{ }_{D T C}\right]=\frac{3 \arctan (t)-3 \arctan (A)-A^{3}+t^{3}+3 A-3 t}{3(\arctan (t)-\arctan (A))}
\end{aligned}
$$

APPENDIX B: MOMENTS

$$
\begin{aligned}
& E(Z)=\frac{1}{C_{(A, B)}}\left[\begin{array}{l}
-4 \alpha\left(A^{2} \alpha+2 B-B^{2} \alpha-2 A+A \alpha^{2}\right)+\frac{1}{12} \alpha^{3}\left(16\left(A^{3}+3 B-B^{3}\right)-3 \alpha(A-B)(A+B)\right. \\
\left.\left(-2+A^{2}+B^{2}\right)\right)+4 \alpha\left(-2+\alpha^{2}\right)(\arctan (A)-\arctan (B))-\frac{1}{2}\left(4-8 \alpha^{2}+\alpha^{4}\right) \log \left[\frac{1+A^{2}}{1+B^{2}}\right]
\end{array}\right] \\
& E\left(Z^{2}\right)=\frac{1}{C_{(A, B)}}\left[\begin{array}{l}
\alpha^{4}(-A+B)+\frac{\alpha^{2}}{15}\left(-3 A^{5} \alpha^{2}+5 A^{3}\left(-8+\alpha^{2}\right)+B^{3}\left(40+\left(-5+3 B^{2}\right) \alpha^{2}\right)\right)+ \\
\left(4-8 \alpha^{2}+\alpha^{4}\right)(\arctan (A)-\arctan (B))+2 \alpha\left(-2+\alpha^{2}\right) \log \left[\frac{1+A^{2}}{1+B^{2}}\right]
\end{array}\right]
\end{aligned}
$$

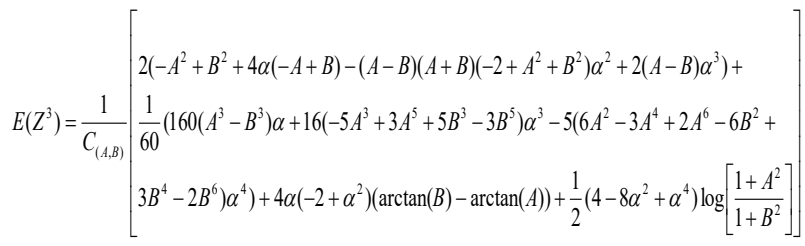

$E\left(Z^{4}\right)=\frac{1}{C_{(A, B)}}\left[\begin{array}{l}4 A-4 B+2 \alpha(A-B)(A+B)\left(-2+A^{2}+B^{2}\right)+8 \alpha^{2}(-A+B)-\alpha^{3}(A-B)(A+B)\left(-2+A^{2}\right. \\ \left.+B^{2}\right)+\alpha^{4}(A-B)+\frac{1}{105}\left(70 A^{6} \alpha^{3}-15 A^{7} \alpha^{4}+21 A^{5} \alpha^{2}\left(-8+\alpha^{2}\right)-35 A^{3}\left(4-8 \alpha^{2}+\alpha^{4}\right)+\right. \\ \left.B^{3}\left(140+56 \alpha^{2}\left(-5+3 B^{2}\right)-70 B^{3} \alpha^{3}+\alpha^{4}\left(35-21 B^{2}+15 B^{4}\right)\right)\right)+\left(4-8 \alpha^{2}+\alpha^{4}\right)(\arctan (B)- \\ \arctan (A))-2 \alpha\left(-2+\alpha^{2}\right) \log \left[\frac{1+A^{2}}{1+B^{2}}\right]\end{array}\right]$ 